\title{
Heliogyro solar sail with self-regulated centrifugal deployment enabled by an origami-inspired morphing reflector
}

DOI:

10.1016/j.actaastro.2018.08.012

\section{Document Version}

Accepted author manuscript

Link to publication record in Manchester Research Explorer

\section{Citation for published version (APA):}

Wu, R., Roberts, P. C. E., Soutis, C., \& Diver, C. (2018). Heliogyro solar sail with self-regulated centrifugal deployment enabled by an origami-inspired morphing reflector. Acta Astronautica. https://doi.org/10.1016/j.actaastro.2018.08.012

\section{Published in:}

Acta Astronautica

\section{Citing this paper}

Please note that where the full-text provided on Manchester Research Explorer is the Author Accepted Manuscript or Proof version this may differ from the final Published version. If citing, it is advised that you check and use the publisher's definitive version.

\section{General rights}

Copyright and moral rights for the publications made accessible in the Research Explorer are retained by the authors and/or other copyright owners and it is a condition of accessing publications that users recognise and abide by the legal requirements associated with these rights.

\section{Takedown policy}

If you believe that this document breaches copyright please refer to the University of Manchester's Takedown Procedures [http://man.ac.uk/04Y6Bo] or contact uml.scholarlycommunications@manchester.ac.uk providing relevant details, so we can investigate your claim.

\section{OPEN ACCESS}




\title{
Heliogyro Solar Sail with Self-Regulated Centrifugal Deployment Enabled by an Origami-Inspired Morphing Reflector
}

\author{
Rui Wu ${ }^{\mathrm{a}, *}$, Peter C.E. Roberts ${ }^{\mathrm{a}}$, Constantinos Soutis ${ }^{\mathrm{b}}$, Carl Diver ${ }^{\mathrm{a}}$ \\ ${ }^{a}$ School of Mechanical, Aerospace and Civil Engineering, the University of Manchester, UK \\ ${ }^{b}$ The University of Manchester Aerospace Research Institute, UK
}

\begin{abstract}
Solar sails utilise solar radiation pressure to propel spacecraft without the need for propellant. Existing solar sail concepts also achieve propellantless flight control by actively twisting the sail or altering the sail surface reflectivity. However, this usually consumes energy and leads to structural dynamic issues. In the present study, we propose an innovative method, which uses origami to convert the 2D sail into a 3D optical meta-structure with design-able and manoeuvrable optical properties. Such a device could adjust both the magnitude and the direction of solar radiation pressure without inducing overall distortion in the sail and therefore achieve flight control through a quasi-static process. As an example, a centrifugally deployed heliogyro solar sail with metastructure morphing reflectors is designed. The reflectors generate stress-dependent solar radiation pressure vector, which propels the spinning of the sail and, according to structural dynamic simulation, achieves a closed-loop controlled centrifugal deployment that is fully passive.
\end{abstract}

Keywords:

photonic propulsion, self-moderating, passive control, gossamer structure, meta material

\section{Nomenclatures}

$a, b, c$ : dimensions of Miura crease pattern

$d$ : length of hinge

$E$ : energy of solar radiation

$f$ : surface reflectivity

$F$ : Force

$l$ : length of folded Miura element

$I$ : angular inertia

$L$ : extension of meta-structure reflector

$m$ : mass

$M$ : moment of force

$n$ : number of reflections

$p$ : momentum of a ray of photon

\footnotetext{
* Corresponding author

Email address: rui.wu@manchester.ac.uk (Rui Wu)
} 
$P_{S R P}:$ solar radiation pressure

$R$ : span of conventional blade

$T$ : tensile load

$U$ : elastic potential energy

$v_{c}$ : speed of light

$W$ : solar radiation energy density

$\alpha$ : surface absorptivity

$\theta$ : dihedral angle (hinge angle) of origami

$\theta_{0}$ : neutral hinge angle

$\epsilon$ : surface emissivity

$\eta$ : edge angle of origami

$\phi$ : Miura angle

$\sigma$ : Stefan-Boltzmann constant $=5.67 \times 10^{-8} \mathrm{~W} \cdot \mathrm{m}^{-2} \cdot \mathrm{K}^{-4}$

$\kappa$ : torsional modulus of $1 \mathrm{~m}$ hinge $(N \cdot \mathrm{m} / \mathrm{rad})$

$\tau$ : torque from hinge

$\omega$ : angular velocity

AoA: Angle of Attack

LEO: Low Earth Orbit

RCD: Reflection Control Device

SRP: Solar Radiation Pressure

\section{Introduction}

Solar sail provides a practical way to achieve propellantless propulsion for a spacecraft using Solar Radiation Pressure (SRP). Efforts towards its technical realisation have started in the 1960s $[1,2,3]$, and various design concepts have been proposed since then. The existing designs can be classified into two categories: rigid sails that achieve structural rigidity from elastic force, and spinning sails rigidised by inertial (centrifugal) force.

Rigid solar sails are formed by attaching reflective membranes onto bracing structures such as deployable booms or spars, which not only constrains the deflection of the membrane when fully deployed, but also initiates and controls the deployment process. Since a large surface area to mass ratio, or low sail loading, is required to maximise the acceleration gained from SRP, it is crucial to minimise structural mass [1]. However, rigid solar sails rely on the elastic bending rigidity of the bracing structures, which makes it disadvantageous and unpractical for large sails due to the high structural mass as well as the buckling limit [4].

Spinning solar sail utilise centrifugal force that is generated from the spinning motion to deploy and stiffen a membrane reflector. Such a design can realise a compression-free structure, eliminating the risk of buckling, and thereby allowing the structure to be thin and flexible. This not only leads to a lower structural mass than the rigid solar sails especially when the sail is large, but also a smaller storage volume when packed, and a lower deployment power consumption [5]. Centrifugal deployment in orbit was first successfully demonstrated in 1993 [6], and the world's first interplanetary solar sail IKAROS launched by JAXA in 2010 was a centrifugally deployed spinning disk sail, which has a square reflector of $14 m \times 14 m$ that is folded and wrapped onto the central spacecraft using a wrap-rib method $[7,8,9]$. Besides spinning disk sails, the concept of heliogyro 
was first introduced in the 1960s [10], and uses multiple centrifugally deployed and stiffened reflective membrane strips to form a helicopter-rotor-like sail. The strips, due to the simple geometry and high slenderness, could be stowed by simply winding around a reel rather than folding along complicated patterns like a disk sail. This allows a higher packing ratio as well as more straightforward packing/deploying process, which make it suitable for solar sails that require large sail area or small storage volume: such as NASA's ambitious Halley Rendezvous sailer designed in the late 1970s with twelve $8 m \times 7.5 \mathrm{~km}$ rectangular blades [11]; or smaller designs such as NASA's HELIOS concept built upon a CubeSat architecture with six $0.75 m \times 220 m$ blades [12]. However, the extreme slenderness $(\sim 1: 1000)$ of a heliogyro blade leads to high tensile stress at the blade root, difficulty in attitude control due to the high blade inertia, as well as risk associated with coupled structural dynamic behaviours $[4,10,13,14]$.

Meanwhile, propellantless flight control of a solar sail propelled spacecraft can be realised by controlling the SRP generated by the sail. In the existing solar sail concepts, this is usually achieved by twisting the reflector, which simultaneously changes the magnitude and direction of the SRP vector. For example, the pitch of different blades of a heliogyro can be controlled in a collective or cyclic manner during each revolution of the spinning motion to achieve spin/thrust control or attitude control like a helicopter [10]. Similarly, the IKAROS disk sail can be divided into multiple panels that are independently pitched to realise spin control [15]. The methods to tilt a sail include directly twisting the sail [10], or generating a pitching moment from SRP by shifting the sail's centre of mass relative to its centre of SRP using moving ballast mass or hinged flaps $[16,17,18]$. Besides those methods that regard the sail as a simple reflector with fixed optical properties, the IKAROS mission has also demonstrated SRP control using Reflection Control Devices (RCD) based on liquid crystal $[7,8]$. The RCDs alter the reflectivity, and thus the magnitude of SRP at certain regions on the sail surface, therefore steer the spacecraft [9]. Similar concepts have also been proposed to control the pitch of heliogyro blades by tip RCDs $[12,19]$. However, RCD manipulates only the magnitude of SRP rather than the direction of it, thus it is still not possible to generate an in-plane thrust component unless the RCD is used to induce overall distortion or displacement in the sail (e.g., when the RCD is used to control the pitch of a Heliogyro blade). On the other hand, the possibility of controlling both the magnitude and direction of the SRP vector, and therefore achieving complex flight control without distorting the whole sail remains to be discovered.

In fact, a better control over SRP vector is viable using origami-based meta-structure with design-able optical properties. Origami provides a method to convert a $2 \mathrm{D}$ sheet such as a solar sail into a 3D device with programmable properties and even built-in functionalities, thus is widely regarded as a building block for meta-materials $[20,21,22,23,24]$. In the present study, a morphing reflector that forms an origami-inspired meta-structure is proposed for spinning sails, which manipulates the SRP vector and thereby can be used to enable a self-regulated centrifugal deployment. The meta-structure is based on a 4-vertex origami [25, 26], which achieves self-regidisation as well as the desired optical properties when folded.

In addition, de-orbiting drag sails, which deploy from a satellite and generate aerodynamic drag in Low Earth Orbit (LEO) to reduce the satellite's orbital altitude and ultimately remove it from the orbit or bring it down for a targeted re-entry, has also attracted interests from the industry [27]. Drag sails have similar design strategies to solar sails since both utilise lightly loaded deployable structures that are large and lightweight [28, 29]. Therefore, the visions and development on solar sails could also benefit drag sail technologies. 


\section{Geometrical properties of the origami-inspired morphing reflector}

The geometry of the proposed meta-structure reflector is based on a 4 -vertex (degree- 4 vertex) origami pattern that contains two types of elements as shown in Figure 1a: Miura element and right-angled zigzag element. The parameters used to describe the folded configuration, including dihedral angles $\theta_{A}, \theta_{Z}$, edge angles $\eta_{A}, \eta_{Z}$, and the length of the Miura element $l$ are shown in Figure $1 \mathrm{~b}$ and $1 \mathrm{c}$. The subscript $A$ and $Z$ denotes the auxiliary line (along x-direction) and zigzag line (along y-direction) respectively.

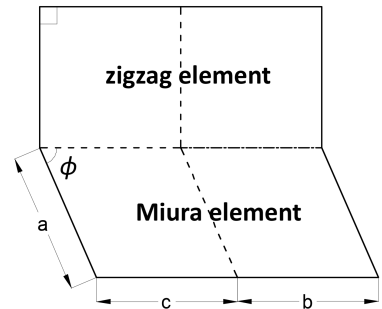

(a)

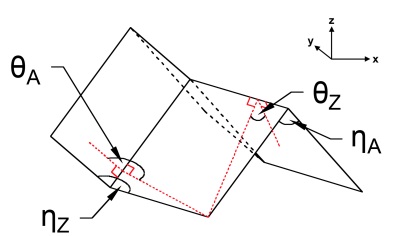

(b)

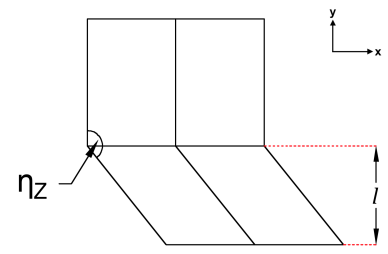

(c)

Figure 1: Parameters of the 4-vertex origami pattern that defines the geometry of the proposed morphing reflector, (a) crease pattern on the flat sail surface containing two types of construction elements, (b)(c) parameters describing a folded configuration

During folding, this origami design has one degree-of-freedom, thus all the dihedral and edge angles are related and depend on each other. The relationships are captured by Eq. 1-3 according to literature [30].

$$
\begin{gathered}
2 \sin ^{2} \eta_{Z}\left(1-\cos \eta_{A}\right)=4 \cos ^{2} \phi \\
\cos \eta_{A}=\sin ^{2} \phi \cos \theta_{Z}-\cos ^{2} \phi \\
\left(2 \sin ^{2} \eta_{Z}-1\right)=\sin ^{2} \phi\left(2 \sin ^{2} \theta_{A}-1\right)+\cos ^{2} \phi
\end{gathered}
$$

Where $\phi$ is a constant for a certain origami design, and once one of the four angles $\theta_{A}, \theta_{Z}, \eta_{A}$ and $\eta_{Z}$ is determined, the rest can be evaluated using Eq. 1-3. Therefore, the origami can morph with one degree-of-freedom. The linear dimensions $a, b$ and $c$ are independent to each other, and can be determined freely.

The overall size of the origami also changes during folding. Particularly, the Miura element's length along y direction can be expressed as below [30]:

$$
l=-a \cos \left(\eta_{Z}\right)
$$

In this origami design, the purpose of the zigzag type element is to generate SRP, while the Miura element controls the folding $\left(\eta_{A}\right)$ of the zigzag element and therefore controls the SRP. The angle between the zigzag elements, which is equal to the edge angle of the Miura elements $\eta_{A}$, is referred to as zigzag angle in the following text. As discussed later in section 3 , when $b \neq c$, an non-zero in-plane (the x-y plane shown in Figure 1) SRP component can be generated under a direct sunlight along z-direction.

The usefulness of origami structures partly comes from its design-ability. In other words, the properties of an origami structure can be adjusted by tailoring the crease pattern or how different 
elements are assembled. The detailed discussions on the design of optical and mechanical properties can be found in Sections 3-4.

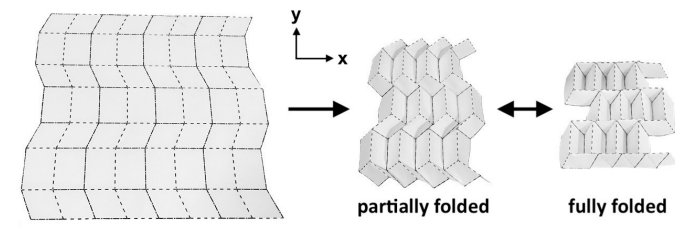

(a)

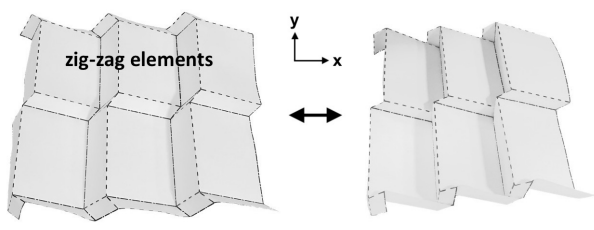

(b)

Figure 2: Paper prototypes of the assembled 4-vertex origami with different design parameters, showing that the origami can morph between the partially and fully folded configurations and the amount of shrinkage can be designed, (a) folding process of a design with $a=b=c$, the overall dimension of which significantly shrinks during folding, (b) a design with $a=b / 2=c / 4$ and extended zigzag elements, which has identical folding angles to (a) but shows much lower shrinkage

Figure 2 shows two examples of assembled origami. It can be seen that the zigzag angle $\eta_{A}$ decreases during folding until the Miura elements are fully folded with $l=0, \theta_{Z}=0^{\circ}$ and $\theta_{A}=\eta_{Z}=$ $90^{\circ}$. Meanwhile, as shown in Figure 2a, the overall area as well as the in-plane linear dimensions ( $\mathrm{x}$ and $\mathrm{y}$ ) of the origami shrinks during folding. However, as suggested by Figure $2 \mathrm{~b}$, this shrinkage can be reduced without influencing the zigzag angle $\eta_{A}$ : by reducing the design parameter $a$ in comparison with $b$ and $c$, by reducing the $b / c$ ratio, or by increasing the relative size of the zigzag elements. A low-shrinkage design allows the optical properties, which depend on the zigzag angle, to vary during folding without inducing significant change in the sail's dimensions and thus avoids structural dynamic problems. The same results can be derived from Eq. 1-4: the angles in the origami $\theta_{A}, \theta_{Z}, \eta_{A}$, and $\eta_{Z}$ are not determined by the length parameters $a, b$ and $c$. Therefore, the shrinkage along y direction, which depends on the dimension of the Miura elements $l$, can be designed independently to the angles and thus the optical properties.

\section{Propulsive properties of the origami-inspired morphing reflector}

According to the discussions above, the optical and geometrical functionalities of the metastructure can be separated into the two types of elements: when the size of Miura element $(a)$ is minor in comparison with zigzag elements, optical properties will almost solely depend on the zigzag elements, while the Miura elements controls folding.

In order to analyse the SRP generated by the zigzag element made from a reflective membrane, a numerical simulator is constructed. It simulates the parallel incident light using 1000 rays equally spaced along the auxiliary line (x-axis), and allows multiple reflections. The evaluation of SRP is based on the momentum transfer between the incident ray and the reflector, as well as between the reflector and the reflected ray. Let the momentum of a ray of photon be $p$, then the force induced on the reflector by the momentum transfer from a incident ray can be calculated by differentiating the momentum by time [1]:

$$
F=\frac{d p}{d t}
$$

According to relativity, we have: 


$$
F=\frac{d E}{v_{c} \cdot d t}
$$

Therefore,

$$
P_{S R P}=\frac{W}{v_{c}}
$$

Similarly, the SRP induced by a reflecting ray is below, note that the direction of $P_{S R P}$ is along the speed of the ray:

$$
P_{S R P}=-\frac{W}{v_{c}}
$$

The analyses are carried out on the 2D cross section of a zigzag element along the auxiliary line. As shown by Figure 3a, the simulation inputs include surface reflectivity, the dimensionless ratio $b / c$, the zigzag angle $\eta_{A}$, and Angle of Attack (AoA), which is the angle between the incident light and the meta-structure's normal direction. It should be noted that the incident light is always in the $\mathrm{x}-\mathrm{z}$ plane since the simulation is $2 \mathrm{D}$. The AoA in the $\mathrm{y}-\mathrm{z}$ plane is not considered since it has the same effect on the zigzag element as on a flat sail. The zigzag angle $\eta_{A}$ changes continuously during folding. For convenience of the simulations, it is varied from the unfolded configuration of $\eta_{A}=180^{\circ}$ to the configuration where the right hand side panel with length $b$ shown in Figure 3 is perpendicular to the sail's central plane. Meanwhile, the AoA is also varied between $\pm 45^{\circ}$.

At the beginning of the simulation, the angle and incident point of each ray is geometrically evaluated using $\eta_{A}$ and AoA. After each reflection, the new incident angle is evaluated using the previous incident angle, and the power density of the ray can be expressed as:

$$
W_{n}=W \times f^{n}
$$

Where $W_{n}$ is the power density of the ray after $n$ times of reflection, and $f$ is the surface reflectivity. The thrust caused by this reflection, $d F_{x}$ and $d F_{z}$ are evaluated using Eqs. 7-8, and then the moment of the thrust, $d M_{y}$ is calculated with respect to the cross-section's centroid. The steps above are repeated until this ray no longer intersects with the origami. During the simulations reported in this paper, the maximum number of reflections encountered by a ray is 3 .

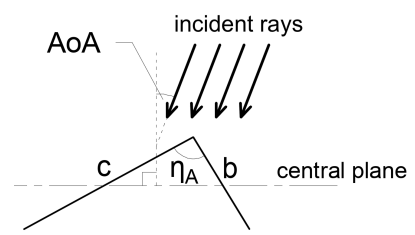

(a)

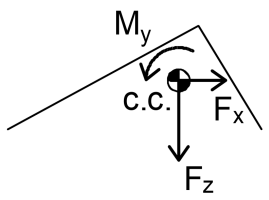

(b)

Figure 3: 2D cross-section of a zigzag element that is simulated with the input parameters shown in (a), and results are shown in (b), where AoA stands for Angle of Attack, and c.c. stands for Cross-section's Centroid

The simulations assume a specular surface reflectivity of $95 \%$, a surface absorptivity of $5 \%$, a solar radiation power density of $1360 \mathrm{~W} / \mathrm{m}^{2}$ (at $1.0 \mathrm{AU}$ ), a sail surface area of $1 \mathrm{~m}^{2}$ when unfolded, and $b+c=1 \mathrm{~m}$. Two meta-structure designs listed in Table 1 are simulated to demonstrate the 
effect of design parameter $b / c$. The results, as shown in Figure $3 \mathrm{~b}$, include the $\mathrm{x}$ and $\mathrm{z}$ components of the force generated from SRP $\left(F_{x}\right.$ and $\left.F_{z}\right)$, as well as the moment about the cross-section's centroid $\left(M_{y}\right)$. Note that the centroid coincides with centre of mass when assuming uniform sail surface density. $M_{y}$ is of interest since it induces twist of the heliogyro blade: due to the blades low torsional stiffness, a small magnitude of $M_{y}$ is required. According to Figure 4 and Table 1, the design objective is achieved: both designs generate a significant in-plane thrust $F_{x}$ without twisting/pitching the sail; and the force can be tuned by simply adjusting the folding angle $\eta_{Z}$ and without inducing significant change of the sail's overall shape or dimensions. This allows the direction of SRP to be adjusted without causing structural dynamic issues. As an example, in section 5 this in-plane thrust is utilised to propel the spinning of a heliogyro.

From Table 1 it can be seen that the two designs yield similar out-of-plane force $F_{z}$, which is the major propulsion that accelerates the spacecraft, while $F_{x}$ and $M_{y}$ can be designed by adjusting $b / c$. It should be noted that the non-zero moment $M_{y}$ about the cross-section's centroid is high enough to induce sail distortion and thus structural dynamic problems. However, since $M_{y}$ is proportional to the chord length of the zigzag element (or in other words, $b+c$ ) multiplies the sail area, it can be reduced without influencing $F_{x}$ and $F_{z}$ by reducing $b+c$ while keeping $b / c$ and the total sail area unchanged (i.e. increasing the number of elements per unit sail area).

Table 1: Simulation results on the SRP-induced forces/moment generated from the zigzag origami element, according to Figure 4

\begin{tabular}{c|c|c|c}
\hline Origami design & Peaks of $F_{x}$ & Peaks of $F_{z}$ & Peaks of $M_{y}$ \\
\hline \multirow{2}{*}{$b / c=0.5$} & $2.95 \mu N$ & $8.83 \mu N$ & $0.92 \mu N \cdot m$ \\
& $-5.00 \mu N$ & $3.62 \mu N$ & $-1.22 \mu N \cdot m$ \\
\hline \multirow{2}{*}{$b / c=0.25$} & $1.78 \mu N$ & $8.83 \mu N$ & $0.40 \mu N \cdot m$ \\
& $-2.65 \mu N$ & $3.96 \mu N$ & $-1.03 \mu N \cdot m$ \\
\hline
\end{tabular}

Besides the results discussed above, according to Figure 5 , a lower $b / c$ leads to a lower dependence of $F_{x}$ on AoA. This could have importance since a lower dependence of $F_{x}$ on AoA leads to lower fluctuation of the in-plane load on the sail during spinning or structural oscillation, and thus a lower tendency of structural dynamic couplings or fatigue. In other words, the determination of $b / c$ leads to a trade-off between the magnitude and the robustness of $F_{x}$.

Furthermore, besides the instantaneous in-plane thrust discussed above, the effective in-plane thrust generated under oscillating AoA is approximated by averaging $F_{x}$ over a period of a harmonic oscillation by time. This represents the AoA fluctuation due to the inclination of the sails spinning axis to the sun or due to harmonic oscillation in the sail structure. 3D analyses are also conducted to account for the effect of AoA in the y-z plane, while the results are very similar and are not reported here. In each subfigure of Figure 6, the five sets of data represented by the five curves assumes different average AoAs, and the amplitude of the AoA oscillation is set to $\pm 20^{\circ}$. As an example, $\mathrm{a} \pm 20^{\circ}$ oscillation with $10^{\circ}$ neutral AoA represents the condition of blades with $10^{\circ}$ constant pitching angle (which can be a result of other types of manoeuvres) and a rotating axis with a $20^{\circ}$ inclination to the sun. By comparing the results to Figure 5, it can be seen that the oscillation has no significant influence on the effective in-plane thrust.

Therefore, it can be concluded that the origami-inspired meta-structure can effectively generate a tuneable in-plane thrust. Such thrust can be robust as the time-average thrust generated by the meta-structure is not significantly influenced by oscillations in AoA (Figure 6), and the effect of a constant AoA can also be limited by adjusting the design parameter $b / c$ (Figure $5 c$ ). 


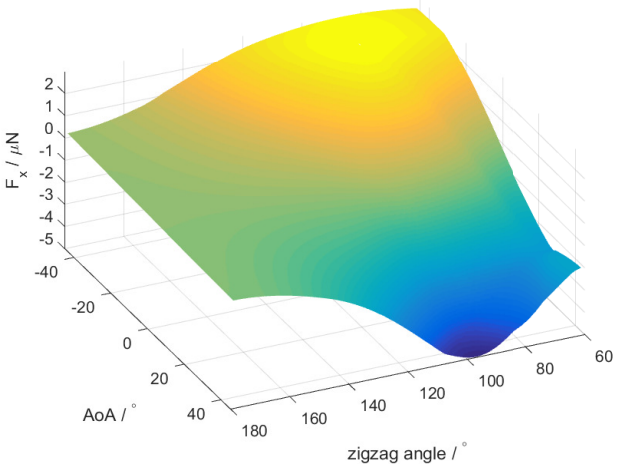

(a)

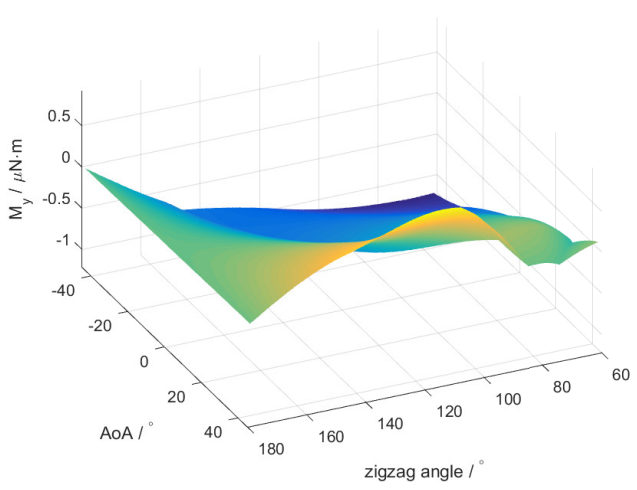

(c)

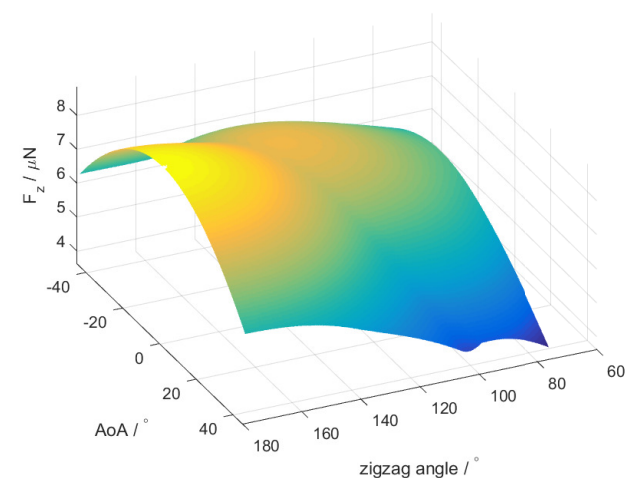

(e)

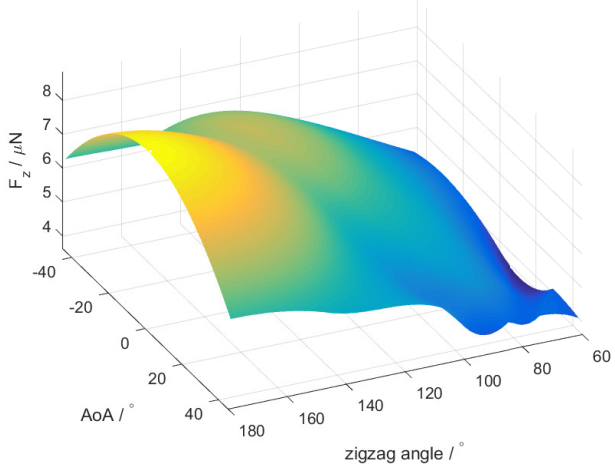

(b)

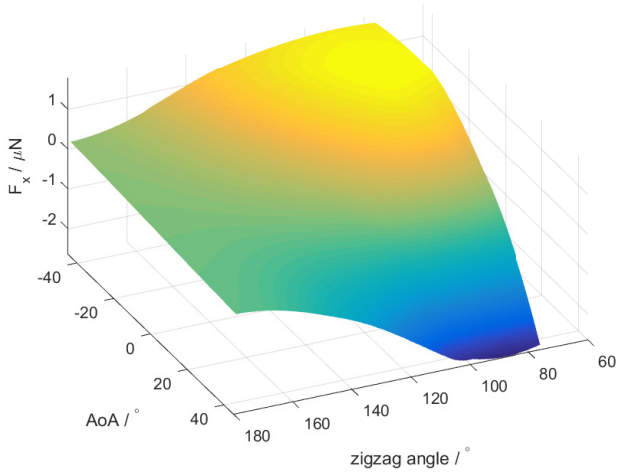

(d)

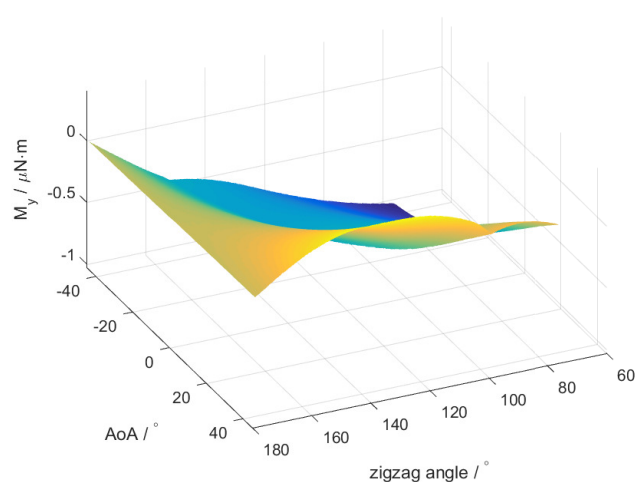

(f)

Figure 4: Simulation results on the SRP-induced forces/moment generated from the zigzag origami element with (a)-(c) $b / c=0.5$, and (d)-(f) $b / c=0.25$, where the torque $M_{y}$ is evaluated by assuming $b+c=1 m$, note that the small fluctuations/discontinuities in the curve are associated with multiple reflections between the origami panels 


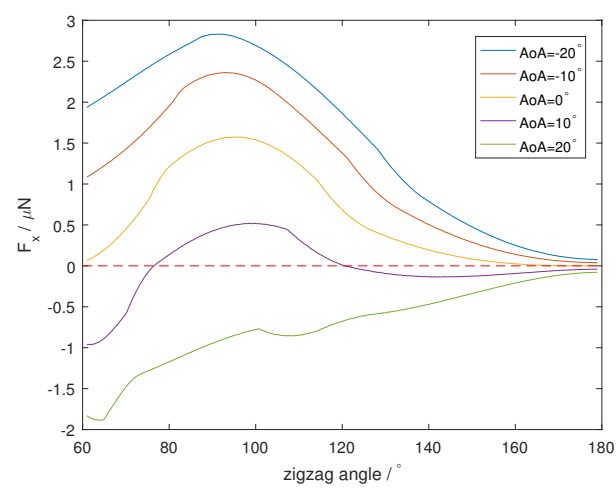

(a)

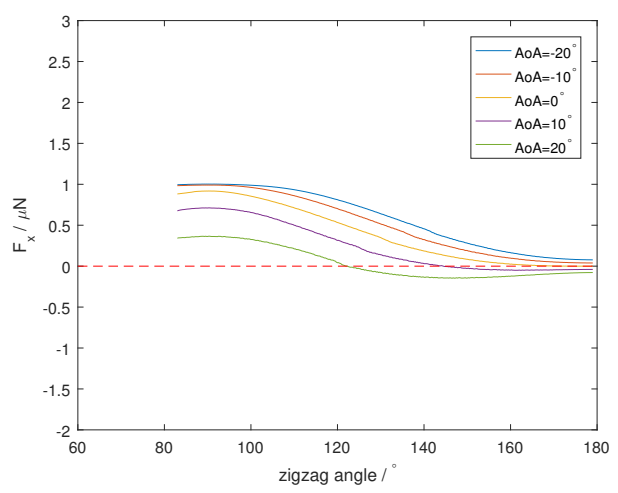

(c)

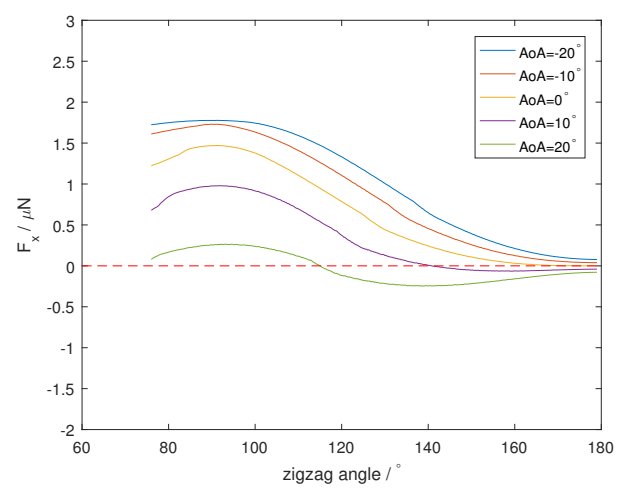

(b)

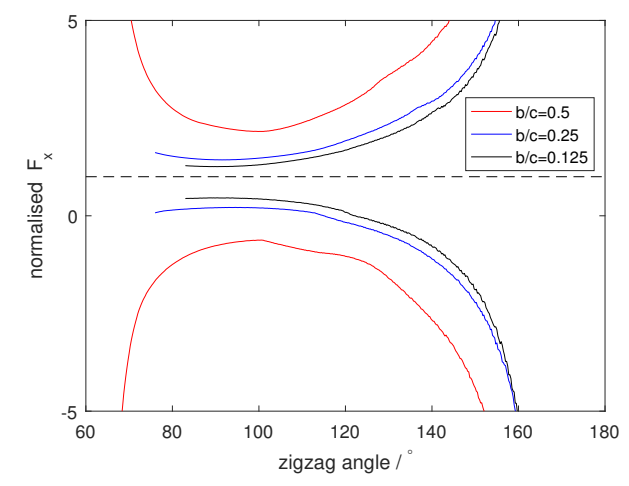

(d)

Figure 5: In-plane thrust $F_{x}$ generated from the zigzag origami element at five different AoAs with (a) $b / c=0.5$, (b) $b / c=0.25$, and (c) $b / c=0.125$, showing that a low $b / c$ ratio gives robust instantaneous in-plane thrusting against AoA oscillation, this is also illustrated in (d), which shows the $F_{x}$ generated at $\pm 20^{\circ}$ AoA normalised by the value at $0^{\circ}$, note that the small fluctuations/discontinuities in the curve are associated with multiple reflections between the origami panels

\section{Mechanical properties of the origami-inspired morphing reflector}

In this section, the morphing reflector's elastic behaviour under a stretching force along $\mathrm{y}$ direction, or in other words, the elastic resistance to shape morphing, is analysed. In the analyses, the reflector is treated as a perfect origami formed by hinged rigid panels, and one basic module as shown in Figure 1 is considered. The hinges are linearly elastic, with the hinges' neutral angle assumed to be $\theta_{0}$ :

$$
\tau=\kappa \cdot\left(\theta-\theta_{0}\right) \cdot d
$$

Therefore, the elastic potential energy of a hinge can be expressed as:

$$
U=\frac{1}{2}\left(\theta-\theta_{0}\right)^{2} \kappa d
$$




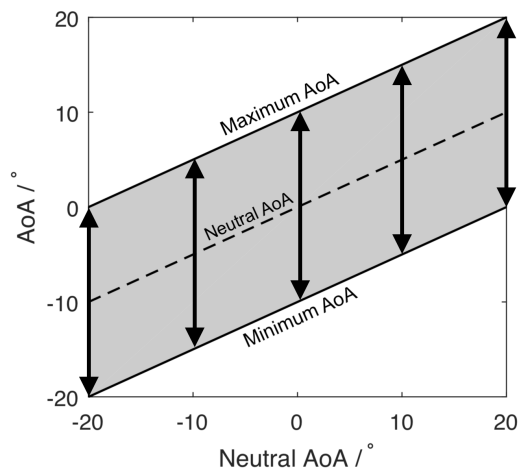

(a)

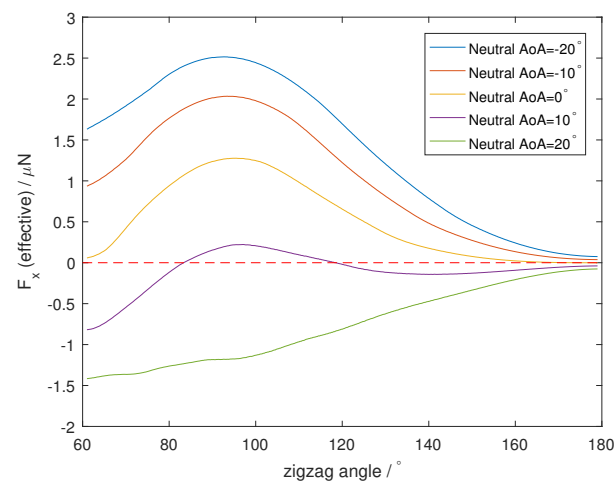

(b)

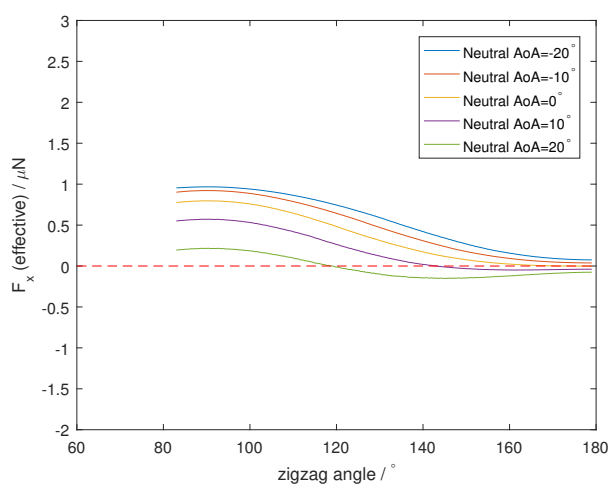

(d)

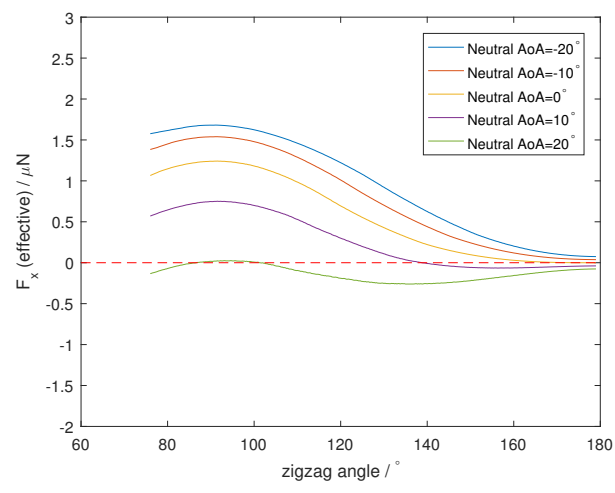

(c)

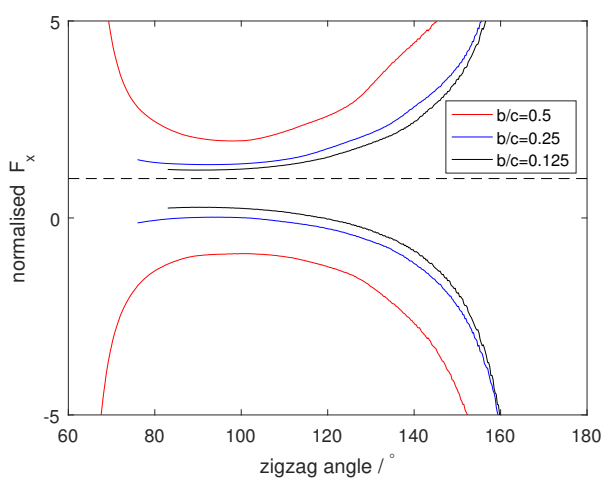

(e)

Figure 6: Time-average of the in-plane thrust generated under oscillating AoAs with five different neutral values and an amplitude of $\pm 20^{\circ}$ as illustrated by (a), with (b) $b / c=0.5$, (c) $b / c=0.25$, (d) $b / c=0.125$; and (e) plots the $F_{x}$ generated at $\pm 20^{\circ}$ AoA normalised by the value at $0^{\circ}$; showing similar results to Figure 5 , thus the effective in-plane thrust is robust against AoA oscillation 
Solving Eq. 1-4, the hinge folding angles in the Miura element, $\theta_{A}$ and $\theta_{Z}$, as well as in the zigzag element, $\eta_{A}$, can be expressed in terms of the origami's length along y-direction $l$. Thus, the elastic potential energy of the whole origami module, $U_{\text {origami }}$ can be expressed in terms of $l$. Then the force reaction along y-direction can be solved by differentiating $U_{\text {origami }}$ with $l$ (the exact expression is not shown here due to its complexity):

$$
F\left(l, a, b, c, d_{z i g z a g}, \phi, \kappa, \theta_{0}\right)=\frac{d U_{\text {origami }}}{d l}
$$

Evaluating $F$ requires the determination of $a, b, c, d_{z i g z a g}, \phi, \kappa$ and $\theta_{0}$. To decide those parameters, a morphing reflector is designed based on NASA's HELIOS concept (Table 2). The design has $b=2.5 \mathrm{~cm}$ and $c=10 \mathrm{~cm}$ to provide an acceptable $b / c$ ratio according to previous discussions. $\theta_{0}$ depends on the initial folding angle of the membrane material, which can be designed to have any value within $0^{\circ} \sim 180^{\circ}$. Here it is set to $90^{\circ}$ to give an example. Based on Figure 5 , in order to achieve a monotonous variation of $F_{x}$ during folding/unfolding and maximise its peak value, $\phi$ is set to $45^{\circ}$ so that $\eta_{A}=90^{\circ}$ when the reflector is fully folded. $\kappa$ is approximated to $2.2 \times 10^{-5} \mathrm{~N} \cdot \mathrm{m} / \mathrm{rad}$ by assuming that the reflector is made of kapton (2.5 GPa young's modulus) with $7 \mu \mathrm{m}$ thickness and the bending of the hinges occurs within a width of $3 \mathrm{~mm}$. Then the elastic force $F$ is evaluated with $a=1 \mathrm{~cm}, 2 \mathrm{~cm}$ and $4 \mathrm{~cm}$ respectively, the results are shown in figure 7. $d_{\text {zigzag }}=100 \mathrm{~cm}$ is chosen here to let the zigzag elements dominate the reflector's propulsive properties.

It can be seen that the elastic property of the reflector is dominated by the zigzag element (in Figure $7, F_{z i g z a g}$ is one order of magnitude greater than $F_{\theta_{A}}$ ), since it colonises the most sail area. Meanwhile, the total force generated by the stretched origami $F_{\text {total }}$, or in other words, the overall resistance to shape morphing, is shown in Figure 8. It can be seen that the elastic resistance to shape morphing can be increased by decreasing $a$ or increasing $d_{z i g z a g}$. According to Eq. 12, this tailoring is a trade-off between the reflector's resistance to morphing and its overall shrinkage/expansion during morphing.

In addition, the resistance to morphing also depends on the way that the origami modules are assembled: setting $n$ modules in parallel (along x-direction) results in a $n$ times of a single module's resistance, while setting modules in series (along y-direction) does not change the resistance.

Meanwhile, as a by-product of turning the 2D membrane into a 3D meta-structure, the flexural rigidity also increases significantly. This is illustrated in Figure 9, where a $6 \mathrm{~cm} \times 25 \mathrm{~cm}$ strip of print paper cantilevered in $1 g$ is set in comparison with a partially folded origami with 195 modules made from a similar strip of paper. Therefore, the shape stability of the meta-structure reflector is expected to be significantly better than a simple film, which could potentially improve the structural dynamic behaviour of a heliogyro. However, the exact effect depends on the detailed hinge design, etc. and is not the scope of the present research.

\section{Heliogyro concept based on the origami-inspired morphing reflector}

As mentioned in introduction, conventional heliogyros utilise helicopter-blade-like flat sails deployed from mandrels to generate SRP. The blade can be pitched by twisting the blade root, shifting the blade centre of mass, or locally adjusting the sail's reflectivity using RCDs. A pitched blade generates an in-plane SRP, thereby achieves attitude manoeuvre and spin control. However, instead of pitching the blade, a in-plane SRP can also be generated using the present meta-structure 


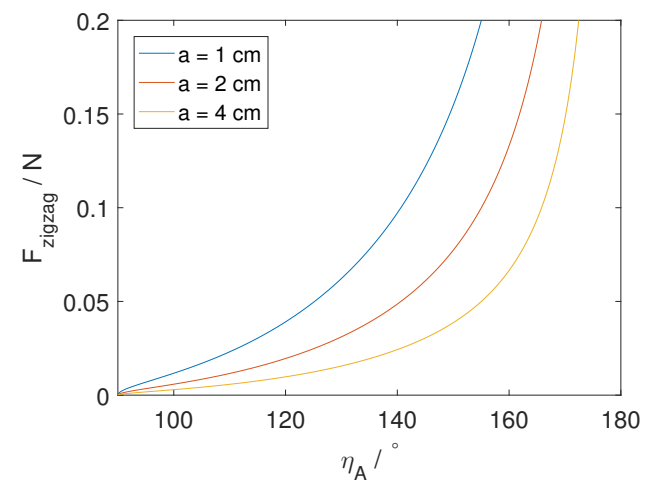

(a)

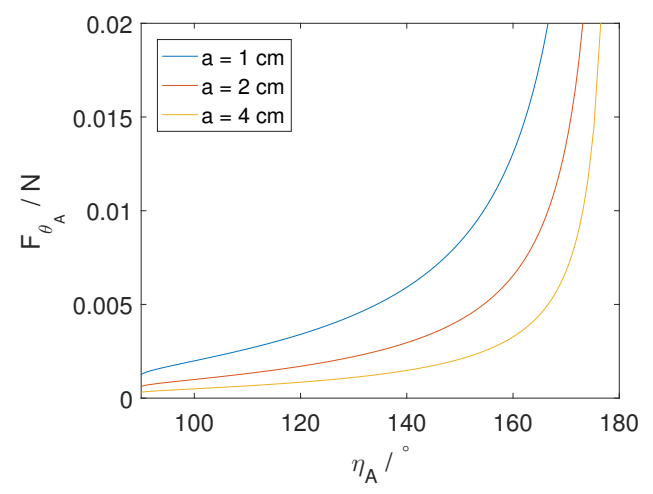

(b)

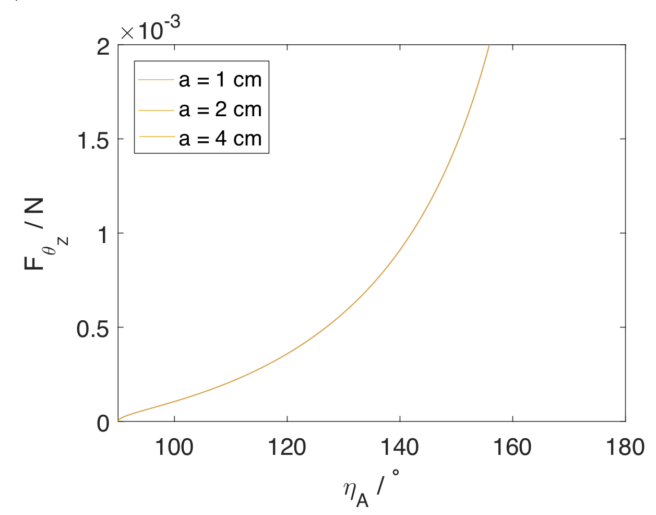

(c)

Figure 7: Elastic recovering force $F$ from an origami module stretched along y-direction, evaluated with three different values of $a$, showing that the elastic property is dominated by the zigzag element (note the difference in axis scaling), (a) force generated from the zigzag element, (b) force generated from the hinges associated with $\theta_{A}$, which is one order of magnitude lower than $F_{z i g z a g}$, (c) force generated from the hinges associated with $\theta_{Z}$, which is two orders of magnitude lower than $F_{z i g z a g}$, note that the three curves overlaps in (c)

morphing reflector. This not only allows the blade to remain in the plane of rotation during manoeuvre and thereby significantly simplifies the blade's structural dynamic behaviour, but also, as demonstrated in this section, realises self-regulating deployment.

In this section, we propose a novel heliogyro blade concept as shown in Figure 10. The blade is consisted of four sections (from left to right): conventional flat heliogyro blade equipped with RCD, meta-structure spin-up reflector, meta-structure braking reflector, and tip mass. The four sections are joined together by transition structures, which allow the morphing of the meta-structure reflectors while transmitting tensile load.

The meta-structure reflectors can be realised using pre-folded polyimide film, which automatically returns to the origami shape when unconstrained. Alternatively, self-folding smart materials could be used to transform a flat reflective membrane into origami when unrolled from the mandrel. However, the material aspect is not within the scope of this article, and remains for future study. 


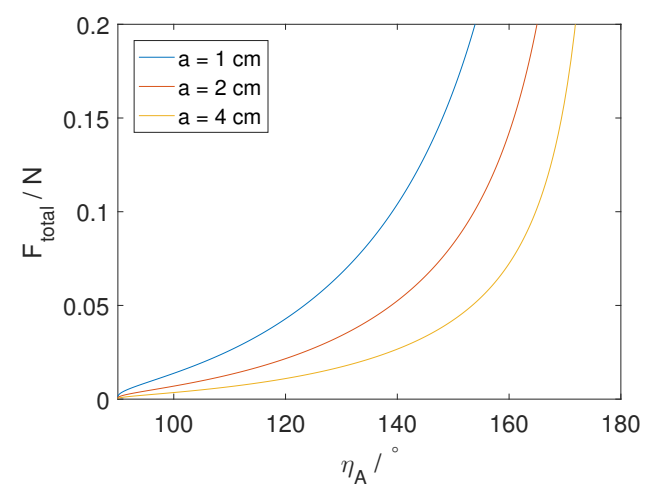

(a)

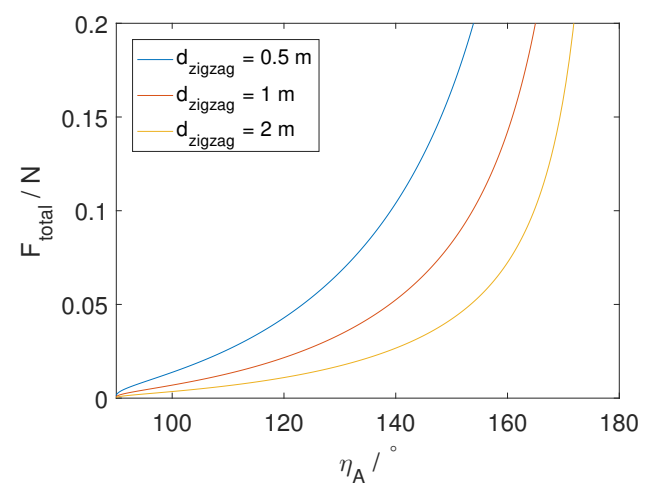

(b)

Figure 8: Total elastic recovering force $F$ from an origami module stretched along y-direction, showing that the elastic property can be tailored, (a) with $d_{z i g z a g}=1 \mathrm{~m}$ and $a$ varied, (b) with $a=2 \mathrm{~cm}$ and $d_{z i g z a g}$ varied

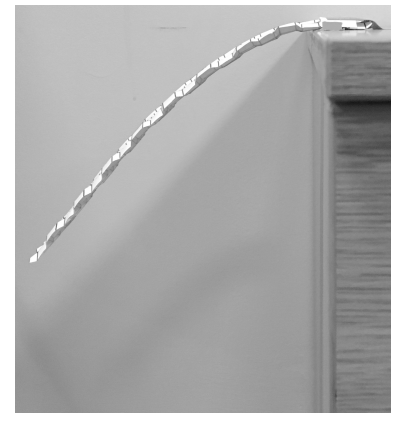

(a)

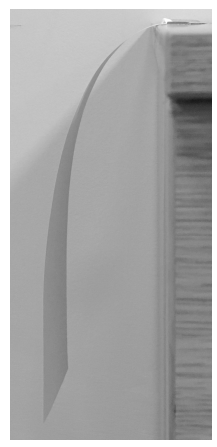

(b)

Figure 9: Besides achieving desired optical and morphing properties, the origami also have higher out-of-plane rigidity after folding, as illustrated by paper prototypes cantilevered in 1g: (a) partially folded origami, (b) flat paper

The function of the meta-structure reflectors are focused on realising self-regulated spin and deployment. Under a static condition as shown in Figure 10, the spin-up reflector generates positive (spin-up) in-plane thrust, which is partially cancelled by the negative in-plane thrust generated by the braking reflector, thus the vehicle will spin-up. At a certain spin rate, centrifugal force stretches the meta-structure reflectors and thereby reduces the in-plane thrusts. However, the tensile strain of the two meta-structure reflectors are different due to the different tensile modulus as well as tensile load. The spin-up reflector, which is designed to have a lower modulus and is under higher tensile load, then generates a much lower spin-up torque that is now fully cancelled out by the negative torque from the braking reflector. Therefore, the spin rate reaches equilibrium under this condition, and a self-regulating spin is achieved. This spin rate will be the final spin rate after the blade is fully deployed.

Meanwhile, as the blade deploys by rolling-off from the mandrel under centrifugal load, the deployment can be passively controlled by the rotational resistance of the mandrel. When the 


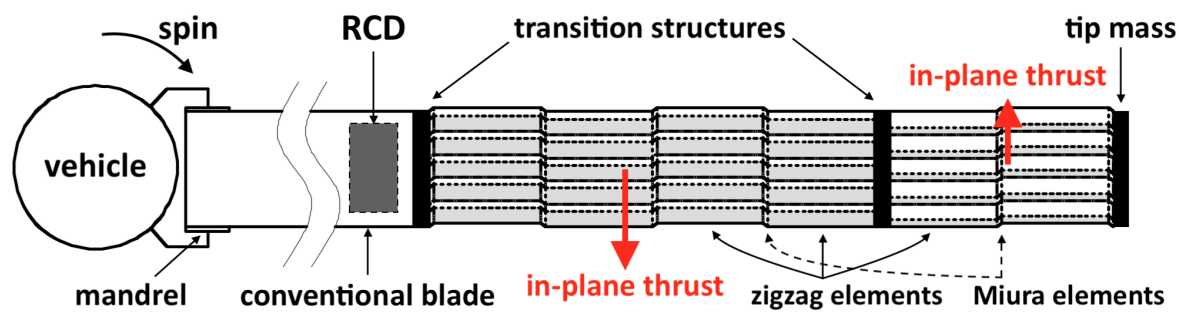

Figure 10: The proposed heliogyro blade concept which achieves passive closed-loop control of centrifugal deployment; the blades is consisted of a conventional flat blade equipped with RCDs, two sections of meta-structure reflectors with different in-plane thrusts and tensile modulus, and a tip mass, joined together by transition structures; note that the vehicle is axisymmetric, though only one blade is shown in this draft

centrifugal force exceeds a threshold, the mandrel will rotate and a small portion of the blade will be released. Release of the blade increases the angular inertia of the whole vehicle, thereby reduces spin rate and centrifugal force. Therefore, the deployment is self-regulated, and the centrifugal force always equals the rotational resistance of the mandrel under an static condition. This offers a way to design a deployment path, or in other words, a spin rate history throughout deployment, by programming the resistance of the mandrel.

Furthermore, the conventional blade is equipped with a RCD in order to realise attitude control. Such RCD is located on the blade centre chord and only effects the magnitude of SRP without inducing pitching or twisting of the blade. Since the aims of the present study is to demonstrate the self-regulated spin and centrifugal deployment enabled by the meta-structure reflectors, the operation of this RCD is not further discussed.

In order to demonstrate the behaviour of this heliogyro with meta-structure reflectors, a sample design based on NASA's HELIOS vehicle is made and the self-regulated deployment is simulated. The parameters of the proposed design, listed in Table 2, are mostly identical or similar to HELIOS. According to the table, both designs have an initial blade length of $20 \mathrm{~m}$ before the main deployment step begins. This initial deployment is carried out while spinning the vehicle up using thrusters, etc. on the vehicle. In the proposed design, all of this pre-deployed $20 \mathrm{~m}$ blade is consisted of the meta-structure reflectors.

The simulations are performed using a Newtonian structural dynamic simulator. It captures the in-plane span-wise motion of the four sections of blade during deployment, as well as the vehicle's spin rate. The simplified model used in the simulation is illustrated by Figure 11. In the model, the blade unrolls from the mandrel, which elastically resists the deployment by the force $T_{\text {root}}$. The meta-structure spin-up reflector is modelled as an homogeneous elastic element, which uniformly elongates under the span-wise tensile load due to the stretch of the origami elements along y-direction. It is worth noticing that in order to achieve a near homogeneous response to the centrifugal-stretch, the span-wise distribution of the origami elements needs to be designed based on the non-uniform tensile stress along the span. This is possible considering the meta-structure's design-able mechanical behaviours. However, the actual design is beyond the scope of the present study. Meanwhile, the meta-structure braking reflector is treated as a rigid panel with constant dimensions and SRP. This is a realistic assumption since the braking reflector is designed to be made of thicker Kapton films in order to prevent elongation and maintain the desired braking SRP force during rotation.

The response of the vehicle's spin rate $\omega$ to the total SRP torque $M_{S R P}$ generated by the blades 
Table 2: Design parameters of HELIOS and the proposed heliogyro [11, 14]

\begin{tabular}{|c|c|c|}
\hline Property & HELIOS design parameters & Proposed design parameters \\
\hline Vehicle mass except blade assembly & \multicolumn{2}{|l|}{$13.3 \mathrm{~kg}$} \\
\hline Vehicle angular inertia except blade assembly & - & $6 \mathrm{~kg} \cdot \mathrm{m}^{2}$ \\
\hline No. of blades & \multicolumn{2}{|l|}{6} \\
\hline Blade chord length & \multicolumn{2}{|l|}{$0.75 \mathrm{~m}$} \\
\hline Full blade length & \multicolumn{2}{|l|}{$220 \mathrm{~m}$} \\
\hline Initial blade length before deployment & \multicolumn{2}{|l|}{$20 \mathrm{~m}$} \\
\hline Length of meta-structure spin-up reflector & - & $18 m$ \\
\hline Length of meta-structure braking reflector & - & $2 m$ \\
\hline Initial spin rate before deployment & $1 R P M[14] \sim 5 R P M[11]$ & $1 R P M$ \\
\hline Final equilibrium spin rate & $1 R P M$ & $1.1 R P M$ \\
\hline Average sail material density & \multicolumn{2}{|l|}{$1.5 \mathrm{~g} / \mathrm{cm}^{3}$} \\
\hline Sail material & \multicolumn{2}{|c|}{$2.54 \mu m$ Mylar $+2 \times 0.1 \mu m$ Al coatings } \\
\hline Spin-up reflector substrate material & - & $7 \mu m$ Kapton $+2 \times 0.1 \mu m \mathrm{Al}$ coatings \\
\hline Braking reflector substrate material & - & $25 \mu m$ Kapton $+2 \times 0.1 \mu m$ Al coatings \\
\hline Blade tip mass & \multicolumn{2}{|r|}{ r } \\
\hline Blade batten mass & \multicolumn{2}{|l|}{$3 \times 3 g=9 g$} \\
\hline Blade assembly mass & $693 g$ & $835 g$ \\
\hline Blade root tensile load when fully deployed & $1 N$ & $1.4 N$ \\
\hline
\end{tabular}

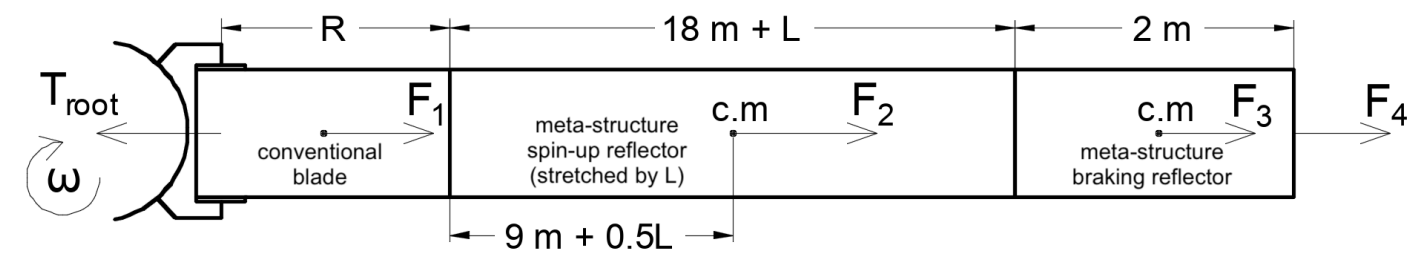

Figure 11: Simplified model for the structural dynamic simulations, where the meta-material spin-up reflector is treated as a homogeneous elastic element that elongates by $L$ under load to simulate the stretch-induced unfolding of the origami, and the meta-material braking reflector is treated as rigid since it is designed to be made of stiffer materials, the inertial forces $F_{1}, F_{2}, F_{3}$ and $F_{4}$ as well as the force from the mandrel $T_{\text {root }}$ are shown in this figure (c.m. stands for centre of mass)

is evaluated as below, where $I$ is the angular inertia of the vehicle including the deployed blades, which changes due to blade deployment and stretch:

$$
\dot{\omega}=\frac{M_{S R P}}{I}
$$

$F_{1}, F_{2}, F_{3}$ and $F_{4}$ are inertial forces from the four sections including the tip mass $\left(F_{4}\right)$. The forces are evaluated using centrifugal acceleration and translational acceleration associated with span-wise motions, and always satisfies:

$$
T_{\text {root }}=F_{1}+F_{2}+F_{3}+F_{4}
$$

Then the mechanical response of the meta-structure spin-up section under a span-wise stretch can be predicted according to Eq. 12:

$$
d U_{\text {origami }}=\left(F_{3}+F_{4}\right) \cdot d l+F_{2} \cdot 0.5 \cdot d l
$$

From this equation, it can be seen that an effective tensile force, which characterises the tension in the spin-up reflector, can be defined as: 


$$
T_{\text {effective }}=\frac{d U_{\text {origami }}}{d l}=0.5 \cdot F_{2}+F_{3}+F_{4}
$$

According to the results in Figure 7, the mechanical properties of the meta-structure reflector is dominated by the zigzag hinges. For simplicity, elastic force from only the zigzag elements is considered in the simulation. The zigzag elements have parameters of $b=2.5 \mathrm{~cm}$ and $c=10 \mathrm{~cm}$ (based on the design in section 4), thus a $0.75 \mathrm{~m}$ blade chord accommodates 6 zigzag elements, which has 11 zigzag hinges. Since the zigzag hinges are aligned along span, which is $18 \mathrm{~m}$ according to Table 2, the total hinge length is then $11 \times 18 \mathrm{~m}=198 \mathrm{~m}$. Then the elastic energy stored in the origami can be evaluated using Eq. 11, and the mechanical response of the spin-up reflector under span-wise tension can be resolved. Combining with SRP analysis results from section 3, Figure 12 is plotted. It can be seen that a zero total SRP spin-up torque is generate when $T_{\text {effective }}=0.39 \mathrm{~N}$, which is the effective tension at the final target spin rate of $1.1 R P M$ under a fully deployed condition $(R=200 \mathrm{~m})$. Therefore, the spin rate will be stabilised at $1.1 R P M$ after deployment. This result requires the sum of the origami parameter $a$ of a series of Miura elements along the spin-up reflector's span to be $12.9 \mathrm{~cm}$. It should be noted that the final equilibrium spin rate $1.1 R P M$ is an asymptote that will never be reached under an ideal condition. It is therefore set to be slightly higher than he final target spin rate of HELIOS $(1 R P M)$ to guarantee that $1 R P M$ can be achieved in a reasonable time.

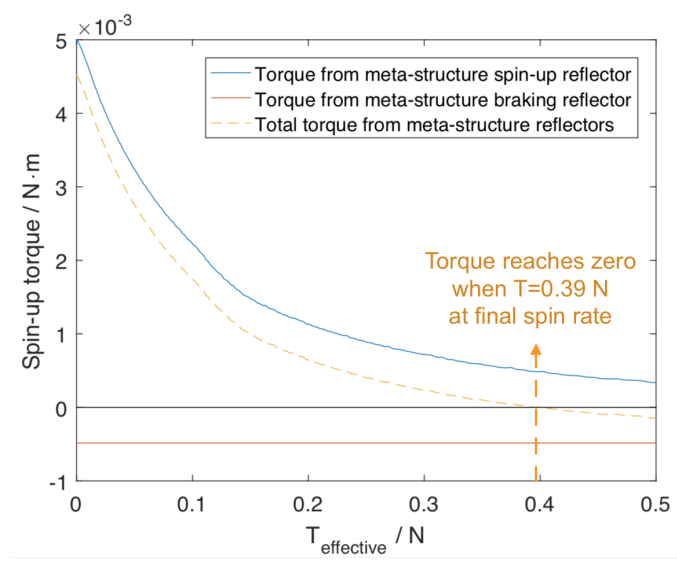

Figure 12: Tension-dependent Spin-up torque generated by the two meta-structure reflectors, which enables the torques to cancel out when reaching the final target spin rate of $1.1 R P M$ and therefore achieve self-regulated spin

As discussed above, the deployment process is controlled by $T_{\text {root }}$, since it determines the centrifugal force threshold beyond which the mandrel starts to rotate and release the blade. Here we assume that $T_{\text {root }}$ comes from a torque-regulating device which connects the mandrel to the spacecraft. The torque from such device can be "programmed" corresponding to the mandrel's angular displacement, thus corresponding to the length of the deployed blade. Two examples are given in Figure 13. The target spin rate history during deployment is designed by "programmming" the centrifugal force threshold, and the simulation results perfectly agree with the design. However, as shown by Figure 14 and Table 3, the two sets of thresholds yield significantly different time to achieve full deployment. In fact, as the vehicle's angular momentum increases during deployment, the rate of deployment is determined by the spin-up SRP torque generated by the meta-structure 
reflectors. Therefore, a rapid deployment such as simulation \#2 needs a high positive in-plane SRP, which, according to Figure 12, requires a low centrifugal force threshold. Meanwhile, Figure 13b and $14 \mathrm{~b}$ also shows that the spin rate grows towards the final (equilibrium) spin rate of $1.1 R P M$ after full deployment is reached.

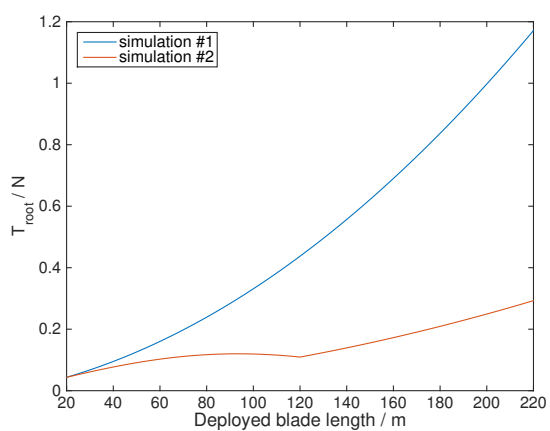

(a)

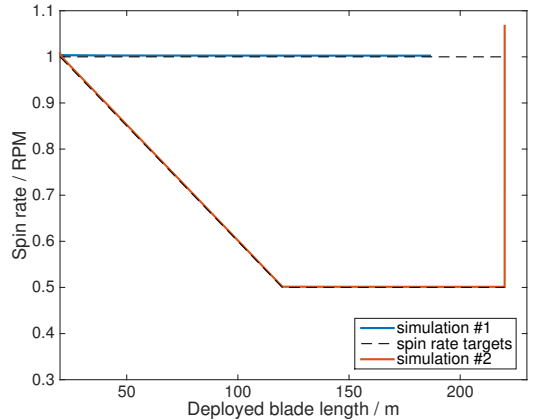

(b)

Figure 13: The simulations use two sets of centrifugal force thresholds, which are "programed" in order to give a certain spin rate history during deployment, where \# 1 gives a constant spin rate during deployment, and \# 2 allows a more rapid deployment, (a) the two sets of centrifugal force thresholds, (b) the simulated spin rate histories, which match the design targets, note that the full deployment of $220 \mathrm{~m}$ is not achieved at the end of simulation \#1 (at day 120), while full deployment is achieved in simulation \#2, after which the spin rate increases toward the final target of $1.1 R P M$

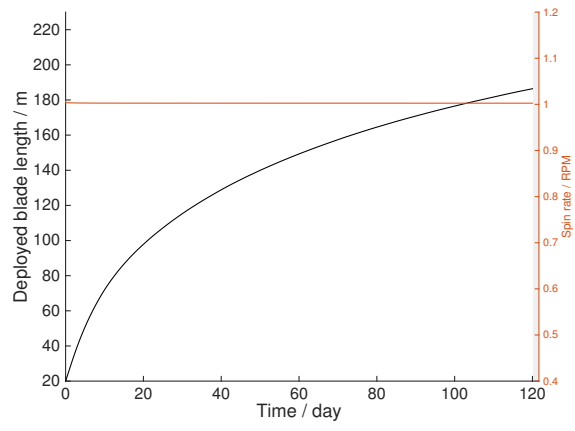

(a)

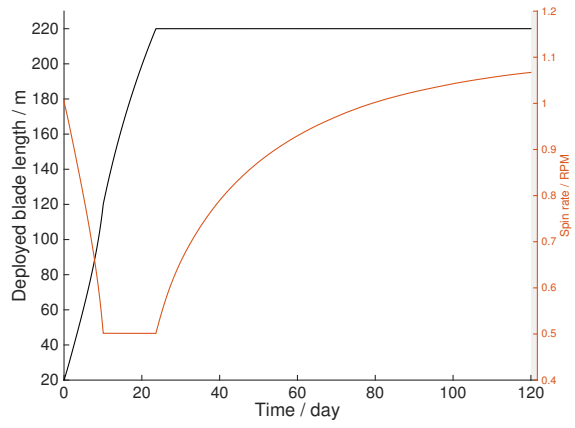

(b)

Figure 14: The simulation results showing the deployed blade length and spin rate histories plotted against time, (a) simulation \#1, in which $186 \mathrm{~m}$ of blade is deployed after 120 days, (b) simulation \#2, in which full deployment is achieved within 24 days

Therefore, as demonstrated by the simulation, by implementing the proposed meta-structure reflectors on the tips of a heliogyro's blades, a vehicle similar to NASA's HELIOS concept can achieve self-regulated spin and passively controlled blade deployment. 
Table 3: Summary of simulation results

\begin{tabular}{c|c|c|c}
\hline Case & Spin rate during deployment & Time to full deployment & Condition at day 120 \\
\hline \hline$\# 1$ & $1 \mathrm{rpm}$ & $>120$ days & $186 \mathrm{~m}$ (partially deployed), $1 \mathrm{rpm}$ \\
\hline$\# 2$ & $0.5 \mathrm{rpm}-1 \mathrm{rpm}$ & $\sim 24$ days & $220 \mathrm{~m}$ (fully deployed), $1.07 \mathrm{rpm}$ \\
\hline
\end{tabular}

\section{Challenges and future work}

Construction of the meta-structure reflector is a critical challenge to the implementation of the proposed design, and opens up opportunities for future innovations. Either as a pre-folded membrane material that automatically returns to the origami shape when unconstrained, or a smart self-folding material that transforms from a flat sheet into a folded origami, the material system has to resist creeping and fatigue and maintain its elastic properties in the harsh space environment.

Another potential challenge is to minimise the shape error in the origami meta-structures and transition structures (see Figure 10), in order to prevent the built-in twist in the reflector. Although the present study has already pointed out that the SRP thrust is generally robust against a varied AoA, the effect of such a twist on the whole sail blade is still unknown. The design of the material system should also consider this effect.

Last but not least, the analyses in this research is based on a simplified mathematical model without considering the deformation of material due to SRP force, or in other words, the solar-elastic effect. Although such effect is weak considering the rigidity of the origami reflector, its influence on the overall shape and the dynamic behaviours of the sail blades remains to be explored.

\section{Conclusions}

A meta-structure solar sail reflector, which has tailor-able geometrical, mechanical and optical properties, is designed based on degree-4-vertex origami. Unlike conventional reflectors that are usually treated as simple reflecting devices, the meta-structure reflector can manipulate the direction and the magnitude of Solar Radiation Pressure (SRP) without changing its orientation to the sun. This could prevent structural dynamic issues of large space membrane reflectors such as heliogyro blades, as it enables flight control without inducing overall distortion (twisting) or out-ofplane motion (pitching) of the sail. Furthermore, the origami folding angles varies under in-plane stretching force in a design-able way, thereby realises a stress-dependent SRP, which can be used to enable a more intelligent system.

As an example, a heliogyro concept with passive self-regulated centrifugal deployment is made, which utilises the stress-dependent in-plane SRP thrust from the meta-structure reflector to propel the spinning motion. During deployment, blades are released from a mandrel, and the behaviour of the vehicle is designed by adjusting the rotational resistance of the mandrel as well as the specific design of the reflector. According to structural dynamic simulations, the spin rate of the vehicle automatically follows the pre-designed deployment path defined by the rotational resistance of the mandrel, and after deployment, the spin rate is regulated by the reflector to prevent over-spin. Using tip-mounted reflectors with $20 \mathrm{~m}$ span on a vehicle similar to NASA's HELIOS design, the full deployment can be achieved within 24 days at $1 A U$.

Therefore, a fully passive heliogyro system with self-regulated centrifugal deployment can be realised using the proposed meta-structure reflector. 


\section{Reference}

[1] B. Fu, E. Sperber, F. Eke, Solar sail technologya state of the art review, Progress in Aerospace Sciences 86 (2016) 1-19.

[2] F. Tsander, The use of light pressure for flight in interplanetary space, problems of flight by jet propulsion, Israel Program for Scientific Translations, Jerusalem, Israel (1964) 303-321.

[3] M. Urbanczyk, Solar sails-a realistic propulsion for spacecraft, Tech. Rep. 67N40314, NASA (1967).

[4] H. Price, J. Ayon, C. Garner, G. Klose, E. Mettler, G. Sprague, Design for a solar sail demonstration mission, in: Space Technology and Applications International Forum, Albuquerque, NM, 2001.

[5] M. Gardsback, G. Tibert, Deployment control of spinning space webs, Journal of guidance, control, and dynamics 32 (1) (2009) 40.

[6] V. Koshelev, V. Melnikov, Large space structures formed by centrifugal forces, CRC Press, 1998.

[7] H. Sawada, O. Mori, N. Okuizumi, Y. Shirasawa, Y. Miyazaki, M. Natori, S. Matunaga, H. Furuya, H. Sakamoto, Mission report on the solar power sail deployment demonstration of ikaros, in: Proceedings of 52nd AIAA/ASME/ASCE/AHS/ASC Structures, Structural Dynamics, and Materials Conference, 2011, pp. 1-11.

[8] Y. Tsuda, O. Mori, R. Funase, H. Sawada, T. Yamamoto, T. Saiki, T. Endo, K. Yonekura, H. Hoshino, J. Kawaguchi, Achievement of ikarosjapanese deep space solar sail demonstration mission, Acta Astronautica 82 (2) (2013) 183-188.

[9] Y. Tsuda, O. Mori, R. Funase, H. Sawada, T. Yamamoto, T. Saiki, T. Endo, J. Kawaguchi, Flight status of ikaros deep space solar sail demonstrator, Acta Astronautica 69 (9) (2011) 833-840.

[10] R. H. MacNeal, The heliogyro-an interplanetary flying machine, Tech. Rep. 67N27627, NASA (1967).

[11] W. K. Wilkie, J. E. Warren, L. G. Horta, K. H. Lyle, J.-N. Juang, J. D. Littell, R. G. Bryant, M. W. Thomson, P. E. Walkemeyer, D. V. Guerrant, et al., Heliogyro solar sail research at nasa, in: Advances in Solar Sailing, Springer, 2014, pp. 631-650.

[12] W. K. Wilkie, J. E. Warren, M. Thompson, P. Lisman, P. Walkemeyer, D. Guerrant, D. Lawrence, The heliogyro reloaded, in: JANNAF Interagency Propulsion Committee Meeting, Huntsville, AL, 2011.

[13] R. S. Blomquist, Heliogyro control, Ph.D. thesis, Carnegie Mellon University (2009).

[14] W. K. Wilkie, J. E. Warren, L. G. Horta, J.-N. Juang, S. C. Gibbs, E. Dowell, D. Guerrant, L. Dale, Recent progress in heliogyro solar sail structural dynamics. 
[15] F. J. Kikuchi, S. T. Chujo, T. Y. Shirasawa, F. O. Mori, Windmill torque estimation of spintype solar power sail with shape control, in: 3rd AIAA Spacecraft Structures Conference, 2016, p. 1218.

[16] P. Wiwattananon, R. G. Bryant, Steering concept of a 2-blade heliogyro solar sail spacecraft, Tech. Rep. NF1676L-24944, NASA (2017).

[17] P. Janhunen, Photonic spin control for solar wind electric sail, Acta Astronautica 83 (2013) 85-90.

[18] P. Janhunen, Electric sail, photonic sail and deorbiting applications of the freely guided photonic blade, Acta Astronautica 93 (2014) 410-417.

[19] D. Guerrant, W. K. Wilkie, D. Lawrence, Heliogyro blade twist control via reflectivity modulation, in: 13th AIAA Gossamer Systems Forum, 2012, pp. 23-26.

[20] Z. You, Folding structures out of flat materials, Science 345 (6197) (2014) 623-624.

[21] C. Lv, D. Krishnaraju, G. Konjevod, H. Yu, H. Jiang, Origami based mechanical metamaterials, Scientific reports 4

[22] L. H. Dudte, E. Vouga, T. Tachi, L. Mahadevan, Programming curvature using origami tessellations, Nature materials 15 (5) (2016) 583-588.

[23] M. Eidini, G. H. Paulino, Unraveling metamaterial properties in zigzag-base folded sheets, Science advances 1 (8) (2015) e1500224.

[24] E. T. Filipov, T. Tachi, G. H. Paulino, Origami tubes assembled into stiff, yet reconfigurable structures and metamaterials, Proceedings of the National Academy of Sciences 112 (40) (2015) $12321-12326$.

[25] H. Fang, S. Li, K. Wang, Self-locking degree-4 vertex origami structures, in: Proc. R. Soc. A, Vol. 472, The Royal Society, 2016, p. 20160682.

[26] H. Fang, S. Li, J. Xu, K. Wang, Locking mechanisms in degree-4 vertex origami structures, in: Proc. SPIE, Vol. 9799, 2016, p. 979910.

[27] P. C. Roberts, P. G. Harkness, Drag sail for end-of-life disposal from low earth orbit, Journal of Spacecraft and Rockets 44 (6) (2007) 1195-1203.

[28] J. Kingston, S. Hobbs, P. Roberts, C. Juanes-Vallejo, F. Robinson, R. Sewell, B. Snapir, J. V. Llop, M. Patel, Use of cypres cutters with a kevlar clamp band for hold-down and release of the icarus de-orbit sail payload on techdemosat-1, Acta Astronautica 100 (2014) 82-93.

[29] V. Melnikova, A. Borovikov, M. Koretskii, Y. Smirnova, E. Timakova, Z. Yu, A. Kuznetsov, K. Frolov, S. Tenenbaum, D. Rachkin, et al., Nanosatellite aerobrake maneuvering device, arXiv preprint arXiv:1708.07417.

[30] J. M. Gattas, W. Wu, Z. You, Miura-base rigid origami: parameterizations of first-level derivative and piecewise geometries, Journal of mechanical design 135 (11) (2013) 111011. 